\title{
Air Fryer using Fuzzy Logic
}

\author{
Abha Tewari \\ Associate Professor \\ V.E.S Institute of Technology
}

\author{
Kiran Israni \\ PostGraduate Student \\ V.E.S Institute of Technology
}

\author{
Monica Tolani \\ PostGraduate Student \\ V.E.S Institute of Technology
}

\begin{abstract}
An air fryer is a kitchen appliance that works by using high speed hot air circulation. When the fryer is working, it begins to produce hot air and a mechanical fan begins to circulate it very fast around the food, which fries the food and produces a crispy layer.

Most hot air fryers come with adjustable temperature and timer knobs that allow for more precise cooking. Food is cooked in the cooking basket that sits on a drip tray. There are various brands of air fryers that claim to fry the food with $80 \%$ less oil in general. Air fryer technology is based on "Rapid air technology" that uses the hot air to fry and cook the food which causes the use of less oil frying food.

Which food needs what amount of cooking time. Time is a business which has not been dealt with properly. In most of the cases the user is compelled with a restricted amount of control. Most of the people wouldn't have noticed (but can reason out very well) different type of food need different amount of cooking time which depends directly on the air temperature and fan speed etc.
\end{abstract}

\section{Keywords}

Fuzzy Logic, Air Fryer, Rapid air technology.

\section{INTRODUCTION}

This paper aims at presenting the idea of controlling the consistency of food using fuzzy logic control. The paper describes the procedure that can be used to get a suitable consistency for different foods. The process is based entirely on the principle of taking non-precise inputs from the sensors, subjecting them to fuzzy arithmetic and obtaining a crisp value of the cooking time. It is quite clear from the paper itself that this method can be used in practice to further automate the air fryer

\section{PROBLEM DEFINITION}

When one uses an air fryer, the person generally sets the timer knob and temperature based on the food type he/she wish to cook and the type and degree of consistency of food. To automate this process, we use sensors to detect these parameters (i.e. temperature, frying time, moisture content, thickness of slice and food consistency). The food consistency is then determined from this data. Unfortunately, there is no easy way to formulate a precise mathematical relationship between temperature, frying time, moisture content, thickness of slice and the food consistency required. Consequently, this problem has remained unsolved until very recently. Conventionally, people simply set timer knob by hand and from personal trial and error experience. Air Fryer is not as automatic as they could be. The sensor system provides external input signals into the machine from which decisions can be made. It is the controller's responsibility to make the decisions. Because the input/output relationship is not clear, the design of an air fryer controller has not in the past lent itself to traditional methods of control design. We address this design problem using fuzzy logic. Fuzzy logic has been used because a fuzzy logic controlled air fryer gives the correct food consistency even though a precise model of input/output relationship is not available.

\section{DETAILS ABOUT THE PROBLEM}

The problem in this paper has been simplified by using four variables.

The four inputs are :

1. Temperature

2. Frying time

3. Moisture content

4. Thickness of slice

Figure (1) shows the basic approach to the problem. The fuzzy controller takes four inputs (as stated for simplification), it processes input and outputs food consistency. Inputs such as temperature and frying time, User will input by using knob. Other two inputs moisture content and Thickness of slice can be left to the sensors (optical, electrical or any type). The working of the sensors is not a matter of concern in this paper. We assume that we have these inputs at our hand. Moisture content of the food and thickness of slice is sensed by the sensor and accordingly it adjusts the fan speed at which the hot air circulates inside the air fryer.

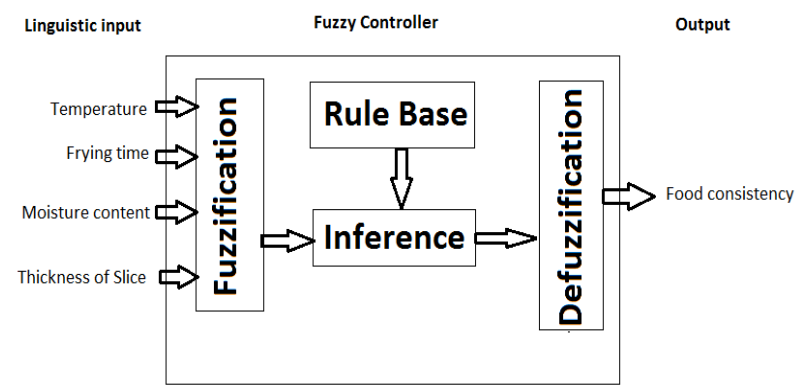

Figure 1 the shape of the membership function

\section{DETAILS ABOUT THE SET APPLIED}

Before the details of the fuzzy controller are dealt with, the range of possible values for the input and output variables are determined. These (in language of Fuzzy Set theory) are the membership functions used to map the real world measurement values to the fuzzy values, so that the operations can be applied on them. Values of the input variables Temperature (range 0 to 200), frying_time (range 0 to 60), 
Moisture_content (range 0 to 100),thickness_of_slice(range 0 to 100) (see figure 2).

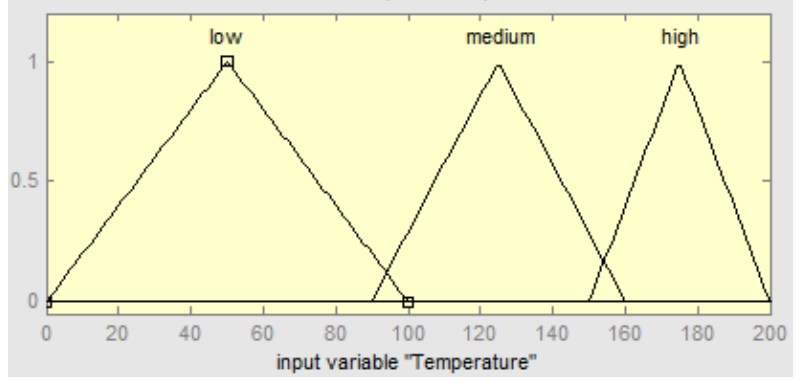

(a)

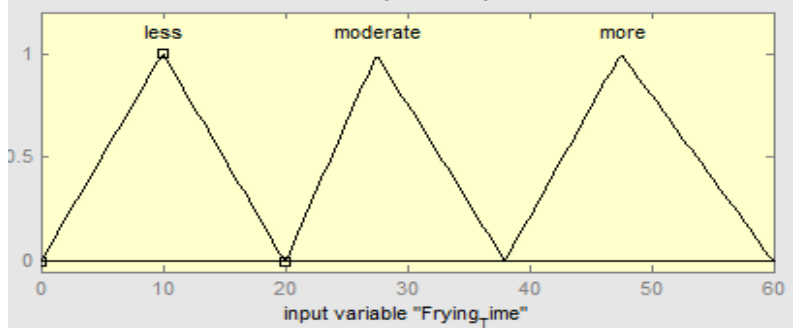

(b)

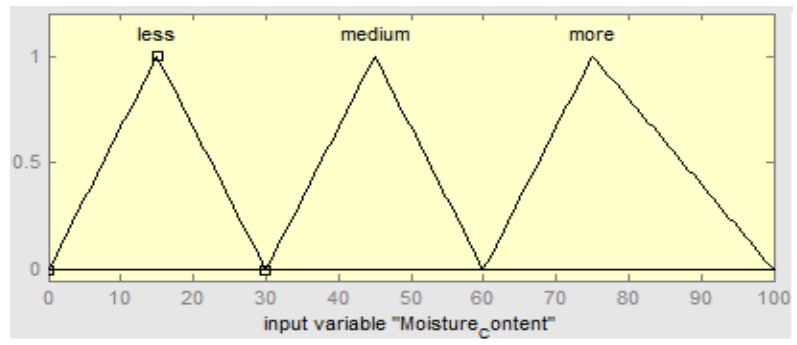

(c)

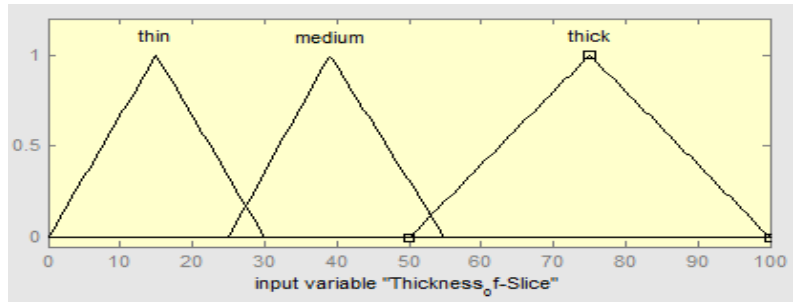

(d)

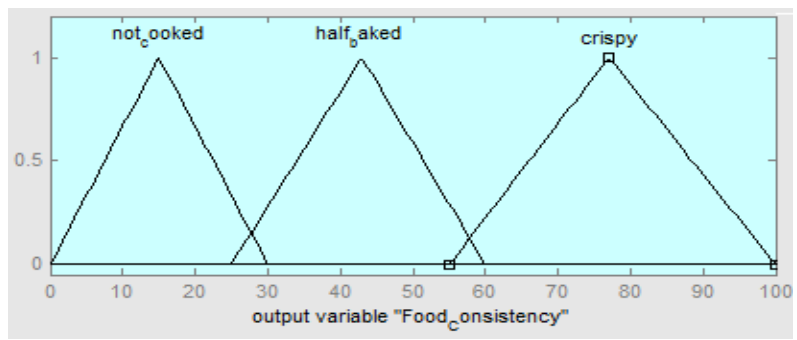

(e)

Figure 2

(a) A membership for input variable Temperatur. (b) A membership for input variableFrying_Time.

(c) A membership for input variable Moisture_content.

(d) A membership for input variable Thickness_Of_Slice.

(e) A memberships for output Food_Consistency.
The decision which the fuzzy controller makes is derived from the rules which are stored in the database. These are stored in a set of rules. Basically the rules are if-then statements that are intuitive and easy to understand, since they are nothing but common English statements. Rules used in this paper are derived from common sense, data taken from typical home use, and experimentation in a controlled environment.

The sets of rules used here to derive the output are:

1. If (Temperature is low) and (Frying_Time is less) and (Moisture content is less) and (Tickness_of_slice is thin) then (Food_consistensy is not cooked);

2. If (Temperature is low) and (Frying_Time is less) and (Moisture_content is medium) and (Tickness_of_slice is thin) then (Food_consistensy is not cooked);

3. If (Temperature is low) and (Frying_Time is less) and (Moisture_content is more) and (Tickness_of_slice is thin) then (Food_consistensy is not cooked);

4. If (Temperature is high) and (Frying_Time is more) and (Moisture_content is medium) and (Tickness_of_slice is medium) then (Food_consistensy is crispy);

5. If (Temperature is high) and (Frying_Time is moderate) and (Moisture_content is more) and (Tickness_of_slice is medium) then (Food_consistensy is half_baked);

6. If (Temperature is high) and (Frying_Time is less) and (Moisture_content is medium) and (Tickness_of_slice is thick) then (Food_consistensy is crispy);

7. If (Temperature is medium) and (Frying_Time is moderate) and (Moisture_content is less) and (Tickness_of_slice is thin) then (Food_consistensy is crispy);

8. If (Temperature is medium) and (Frying_Time is less) and (Moisture content is more) and (Tickness_of_slice is thick) then (Food_consistensy is not cooked);

9. If (Temperature is low) and (Frying Time is less) and (Moisture content is more) and (Tickness_of_slice is medium) then (Food_consistensy is not cooked);

10. If (Temperature is high) and (Frying_Time is more) and (Moisture content is less) and (Tickness_of_slice is medium) then (Food_consistensy is crispy);

The rules too have been defined in imprecise sense and hence they too are not crisp but fuzzy values. (See figure 3 ) The four input parameters are fuzzified as per the membership function of the respective variables. These in additions with the membership function curve are utilized to come to a solution (using some criteria). At last the crisp value of the food_consistency is obtained as an answer. 


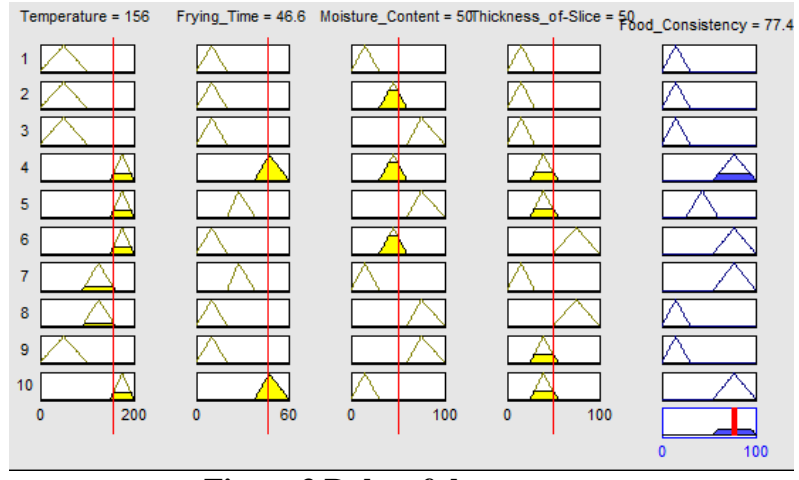

Figure 3 Rules of the system.

In this paper we use a FUZZY TOOLBOX in MATLAB software program to present our model system. This tool allowed us to justify inputs and outputs depend on our own design. So in (figure $2 \mathrm{a}, \mathrm{b}, \mathrm{c}$ and d) you can see that we apply triangular membership for input permeates and we get the resulted output as seen in (figure 2 , e).

\section{RESULTS AND DISCUSSION}

The sensors sense the input values and using the above model the inputs are fuzzy field and then by using simple if-else rules and other simple fuzzy set operations the output fuzzy function is obtained and using the criteria the output value for food consistency is obtained. Figure 4 shows the response surface of the input output relations as determined by Fuzzy Interface Unit. This is the fundamental unit in which the application interface encodes controller information.

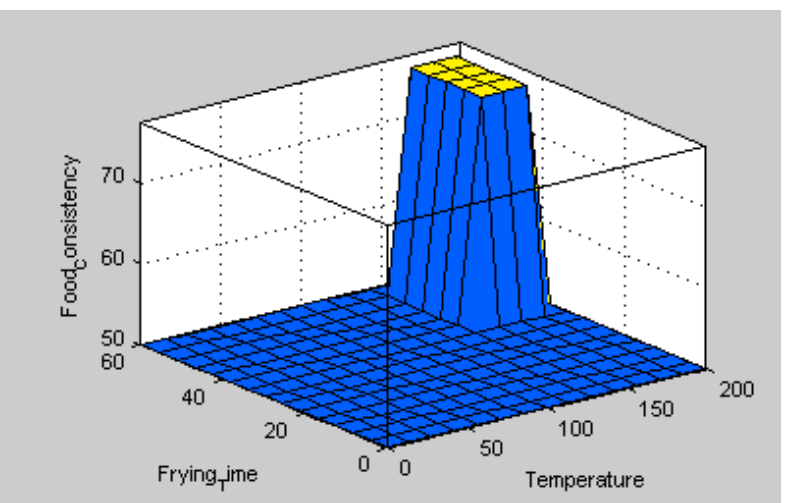

Figure 4 Response surface of the input output relations. The results (the above plot) shows the way the air fryer will response in different conditions. For example, if we take temperature high, frying time as more, Moisture content as medium and thickness of slice as medium Then food consistency which the model output is crispy.

\section{MODEL AND CALCULATION}

Like a real Air Fryer would, our model first tests what is the moisture content and thickness of slice. Once it knows all the parameters, it can easily calculate consistency of food.

First it always takes a base of 10 minutes. It does this so that people are happy with its work even if they put completely cooked food. It then calculates to what degree the food is uncooked If it is $100 \%$ uncooked it adds two minutes per piece of food. Of course a real air fryer would just do these calculations in the end, but our model does it for each individual piece so you can keep track of what is going on easier.
So if you now add a piece which is only $50 \%$ cooked, it will add $50 \%$ of 2 minutes; it adds 1 minute instead of 2 minutes to the base of 10 minutes. Our air fryer, however, doesn't only check for uncooked food but also for moisture content.

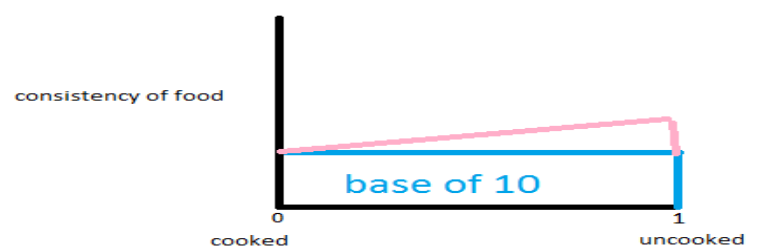

Figure5 (a)

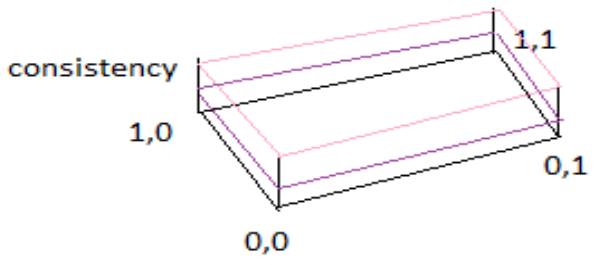

Figure5 (b)

Figure 5 (a) Choosing base time as reference. 5(b) Relation between base time and the system inputs

\section{SUMMARY}

By the use of fuzzy logic control we have been able to obtain a consistency of food for different temperature, frying time, moisture content and thickness of slice. The conventional method required the human interruption to decide upon what should be the cooking time for different foods. In other words this situation analysis ability has been incorporated in the machine which makes the machine much more automatic and represents the decision taking power of the new arrangement.

Though the analysis in this paper has been very crude, but this clearly depicts the advantage of adding the fuzzy logic controller in the air fryer. Also we do interface to validate our model. This interface makes the system easy to deal with it for user and any interested in this sector of engineering.

\section{FUTURE DIRECTIONS}

A more fully automatic air fryer is straightforward to design using fuzzy logic technology. Moreover, the design process mimics human intuition, which adds to the ease of development and future maintenance. Although this particular example controls only the consistency of food of a air fryer, the design process can be extended without undue complications to other control variables. The formulation and implementation of membership functions and rules is similar to that shown for consistency of food.

\section{REFERENCES}

[1] Technical manual of Air Fryer, Philips,at ( http://www.p4c.philips.com).

[2] H. Wu, T.G. Karayiannis*, S.A. Tassou, A twodimensional frying model for the investigation and optimisation of continuous industrial frying systems, october 2012

[3] P. Singhala, D. N. Shah, B. Patel ,Temperature Control using Fuzzy Logic,january 2014 
[4] Bart Kosko, Fuzzy Logic, Scientific American journal, July 1993.

[5] Designing with FuzzyLogic, IEEE Spectrum,November 1990Designing with Fuzzy

[6] Fuzzynet technical case studies (http://www.aptronix.com)
[7] Fuzzylogic,(http://mathematica.ludibunda.ch/fuzzyl ogic6.html)

[8] https://en.wikipedia.org/wiki/Air_fryer 